\title{
Article
}

\section{Trajectories of university adjustment in the United Kingdom: Emotion management and emotional self-efficacy protect against initial poor adjustment}

Nightingale, S.M., Roberts, S, Tariq, Vicki, Appleby, Yvon, Barnes, Lynne, Harris, Rebecca, Dacre-pool, Lorraine and Qualter, Pamela

Available at https://clok.uclan.ac.uk/8988/

Nightingale, S.M., Roberts, S, Tariq, Vicki, Appleby, Yvon, Barnes, Lynne orcid iconORCID: 0000-0003-4504-7139, Harris, Rebecca orcid iconORCID: 00000003-4326-2425, Dacre-pool, Lorraine orcid iconORCID: 0000-0003-20498670 and Qualter, Pamela (2013) Trajectories of university adjustment in the United Kingdom: Emotion management and emotional self-efficacy protect against initial poor adjustment. Learning and Individual Differences, 27 . pp. 174-181. ISSN 1041-6080

It is advisable to refer to the publisher's version if you intend to cite from the work. http://dx.doi.org/10.1016/j.lindif.2013.08.004

For more information about UCLan's research in this area go to http://www.uclan.ac.uk/researchgroups/ and search for <name of research Group>.

For information about Research generally at UCLan please go to http://www.uclan.ac.uk/research/

All outputs in CLoK are protected by Intellectual Property Rights law, including Copyright law. Copyright, IPR and Moral Rights for the works on this site are retained by the individual authors and/or other copyright owners. Terms and conditions for use of this material are defined in the policies page. 
Title: Trajectories of university adjustment in the United Kingdom: Emotion management and emotional self-efficacy protect against initial poor adjustment.

Nightingale, S.M. , Roberts, S. ${ }^{\text {* }}$, Tariq, V. , Appleby, Y. ${ }^{\mathrm{d}}$, Barnes, L. ${ }^{\mathrm{d}}$, Harris, R.A. , DacrePool $^{\mathrm{a}}$ \& Qualter, P. ${ }^{\mathrm{a}}$

${ }^{\mathrm{a}}$ School of Psychology, ${ }^{\mathrm{c}}$ School of Social Work, and ${ }^{\mathrm{d}}$ School of Education and Social Science, University of Central Lancashire, Preston, PR1 2HE, UK

${ }^{\mathrm{b}}$ School of Education, University of Leeds, Leeds, LS2 9JT, UK

* Corresponding author: Dr. Sian Roberts. School of Education, University of Leeds, Leeds, LS2 9JT, UK.

Telephone: +44 (0)1133434601.

E-mail address: S.Roberts@leeds.ac.uk 


\begin{abstract}
Little is known about individual differences in the pattern of university adjustment. This study explored longitudinal associations between emotional self-efficacy, emotion management, university adjustment, and academic achievement in a sample of first year undergraduates in the United Kingdom $(\mathrm{N}=331)$. Students completed measures of adjustment to university at three points during their first year at university. Latent Growth Mixture Modeling identified four trajectories of adjustment: (1) low, stable adjustment, (2) medium, stable adjustment, (3) high, stable adjustment, and (4) low, increasing adjustment. Membership of the low, stable adjustment group was predicted by low emotional self-efficacy and low emotion management scores, measured at entry into university. This group also had increased odds of poor academic achievement, even when grade at entry to university was controlled. Students who increased in adjustment had high levels of emotion management and emotional self-efficacy, which helped adaptation. These findings have implications for intervention.
\end{abstract}

Keywords: Emotional intelligence; emotional management; emotional self-efficacy; trajectories; academic achievement; university adjustment; growth mixture modeling. 


\section{Introduction}

Self-reported poor adjustment to university is the main factor predicting student attrition and low academic results (Dahmus, Bernardin, \& Bernardin, 1992; Gerdes \& Mallinckrodt, 1994; Prillerman, Myers, \& Smedley, 1989; Rickinson \& Rutherford, 1995, 1996; Sennett, Finchilescu, Gibson, \& Struass, 2003; Strahan, 2003). Among the typical markers of maladjustment are loneliness, depression, unhappiness, dissatisfaction, and perceived stress (Beyers \& Goossens, 2002). There are many studies examining adjustment to university, but there are two major gaps in our knowledge. First, the majority of research includes US students, and little is known about adjustment to university in the UK. Given the cultural differences regarding student entry into universities in the UK and US (Weko, 2004), we should not assume that transition to university is the same for students in the UK and US. Second, although research has examined the time course of adjustment to university, there is a scarcity of research examining individual differences in patterns of adjustment over time. The current study redressed these oversights by examining (1) adjustment for a cohort of first-year students in a British university, (2) the different patterns of adjustment to university, (3) the predictors of groups following different trajectories of adjustment to university, and (4) whether following particular trajectories of adjustment puts students at risk of withdrawal from university and academic underachievement.

\subsection{Trajectories of adjustment over time}

Prospective cohort studies have found the first year is a difficult time for university students (Cooke, Bewick, Barkham, Bradley, \& Audin, 2006; Gall, Evans, \& Bellerose, 2000; Peterson, Louw, Dumont, \& Malope, 2010; Pritchard, Wilson, \& Yamnitz, 2007; Soucy \& Larouse, 2000; Tao, Dong, Pratt, Hunsberger, \& Pancer, 2000). However, patterns of adjustment presented in these empirical studies are different. Cooke and colleagues, for example, reported a 'U' shape of adjustment, with students started the first year with high 
well-being, but deteriorated towards the middle of the year, recovering by the end of that first year at university. Other research (Gall et al.) found a pattern characterized by poor adjustment at the start of year 1, with gradual improvement over the course of that year. Pritchard and colleagues found that adjustment decreased over the first year, with undergraduates’ psychological and physical health declining. There are many reasons why different patterns are found between university cohorts, but one possibility is that not everyone within the student population is responding and behaving in the same way.

These studies yielded important information about trends in adjustment, but they examined only mean adjustment scores. Work on mental health suggests great variability in people’s ability to cope over time (Galatzer-Levy, Burton, \& Bonanno, 2012) and it is likely that within any given population, there will be different patterns of adjustment. The previous studies examined predictors of individual differences in adjustment, but this does remove the fact that the statistical methods used averages across subgroups of individuals who may follow different courses of development. Relatively new analytical methods, such as latent class growth analysis modeling (LCGM: Nagin, 1999) and latent growth mixture modeling (LGMM: Muthén, 2004), allow us to examine differences in the time course of adjustment, providing more accurate information about distinct subgroups. These techniques provide the means to examine different patterns of adjustment across the first year, which enables the development of interventions that address the specific needs of individual students.

Using LCGM, Duchesne and colleagues (2007) identified different adjustment trajectories of science students in the US from the end of high school to the end of their second year at university. Their findings indicated that not all university students followed the same course of adjustment, with some students following a high stable trajectory of adjustment and others following a declining trajectory of adjustment; the adjustment path followed was predicted by specific earlier information, notably gender and parental 
involvement at the end of high school. However, other comprehensive studies that investigate these developmental trajectories across different samples are needed. Such an examination can identify protective factors against poor adjustment. In the current study, we examined (1) individual patterns of adjustment across the first year of university with a sample of students in a UK university, (2) the role of emotional intelligence in predicting adjustment trajectory, and (3) whether membership of particular adjustment trajectory groups predicted academic success and first-year retention.

\subsection{Adjustment to university: The importance of Emotional Intelligence}

EI is conceptualised in two ways: (1) as an emotion-related cognitive ability involving the ability to perceive, use, understand, and regulate emotion (Mayer, Salovey, \& Caruso, 2004), and (2) as behavioural dispositions and self-perceptions of one’s ability to recognize and understand emotions (see Petrides, Pita, \& Kokkinaki, 2007). These two perspectives have been termed ability EI (AEI) and trait EI (TEI) respectively.

AEI and TEI have been linked empirically to adaptive functioning (see review by Brackett, Rivers, \& Salovey, 2011); both promote positive ways of coping with stressful situations (Davis \& Humphrey, 2012), which leads to adaptation (Keefer, Parker, \& Saklofske, 2009). Davis and Humphrey argue that AEI drives the appropriate selection of coping strategies, whilst TEI determines coping efficacy. Within education, AEI should influence the complicated cognitive procedure of picking the correct coping strategy in response to academic and social stressors, and the accurate follow-through in terms of actual behavioral response. AEI also impacts the use of ongoing feedback so that the person makes changes to that coping response. TEI is important for university adjustment primarily because emotional self-efficacy is an important aspect of that construct (Petrides, Furnham, \& Mavroveli, 2007; Petrides, Pita, \& Kokkinaki, 2007), which should influence how students persevere in the face of academic difficulties and how resilient they are to academic stressors. 
Building on previous empirical work on the influence of self-efficacy and skills knowledge (e.g. Bandura, 1986), both TEI, specifically emotional self-efficacy, and AEI should have independent effects on academic outcomes because there are differences between beliefs about the ability to perform a behavior and actually performing that behavior.

In support of this notion, amongst university samples, AEI and TEI have been shown to provide an adaptive advantage predicting better adjustment. AEI predicts academic success, even after personality and academic intelligence are statistically controlled (Lyons \& Schneider, 2005). The AEI facet of emotion management is particularly important in determining whether students cope with adaptation to specific and general university stressors (MacCann et al., 2011; Tariq et al., 2013). TEI also predicts academic achievement of university students (e.g., Austin, Evans, Goldwater, \& Potter, 2005; Parker, Summerfeldt, Hogan, \& Majeski, 2004), first-year undergraduate retention (Parker, Hogan, Easterbrook, Oke, \& Wood, 2006; Qualter, Whiteley, Morley, \& Dudiak, 2009) and multi-year retention rates (Keefer, Parker, \& Wood, 2012). TEI also impacts successful social adjustment at university (Engelberg and Sjoberg, 2004), which in turn can aid adjustment (Christie, Munro, \& Fisher, 2004).

These findings indicate that undergraduate students with greater EI use more effective coping strategies for academic and social situations (Austin, Saklofske, \& Mastoras, 2010; Saklofske, Austin, Galloway, \& Davidson, 2007). Skills in managing emotion are most consistently implicated in these associations (Davis \& Humphrey, 2012; Tariq et al. 2013; MacCann, Fogarty, Zeidner, and Roberts, 2011).

\subsection{The current study}

In the current study, we used latent growth mixture modeling techniques to examine different developmental trajectories of adjustment. The College Adaptation Questionnaire (CAQ: Crombag, 1968) was used to measure adjustment because it correlates negatively with 
loneliness, depression and interpersonal helplessness, and positively with life satisfaction at university (van Rooijen, 1986); it has been successfully used in UK studies (e.g. Baker, 2004; Swanson et al, 2006). Based on previous work by Duchesne et al. (2007) we expected to find distinct trajectories of adjustment to university. However, because LCGM and LGMM are sample-specific, the number and nature of trajectories found in the current study may not exactly replicate those of Duchesne et al. To provide validation of the adjustment groups, two further measures of psychological adjustment (measures of loneliness and depressive symptoms) were used.

AEI and TEI have been shown to be important for adjustment to university, but there is no examination of how they might predict different patterns of university adjustment over time. We were interested in the role of emotional self-efficacy (ESE), a part of TEI, and the emotion management facet of AEI in this association because the theoretical frameworks of AEI and TEI propose different roles for emotion management and ESE in predicting university adjustment. Important for our examination is evidence showing (1) skill in managing emotions predicts adaptation (Davis \& Humphrey, 2012; MacCann et al., 2011) and (2) emotional self-efficacy (ESE), a dimension of TEI, influences behaviour (e.g. Bandura, 1986, 2003; Dacre-Pool \& Qualter, 2012a; Kirk et al., 2008). Also of importance is empirical work showing each TEI dimension should be considered separately in analyses, with students high on at least one TEI dimension having better outcomes than students who have no individual TEI strength (Keefer et al., 2012). Thus, we examined how the combined AEI (tested only in terms of emotion management) and TEI (assessed only in terms of ESE) profiles predict trajectory of adjustment. The identification of predictors of positive or poor adjustment can be used to determine which students would benefit from specific interventions. 
As part of our analyses, we examined whether students following particular adjustment trajectories had low academic performance and first-year drop-out. Based on previous empirical work, we expected students who reported poor adjustment to university in year 1 (poor, stable or dropping levels of adjustment) to have poorer grades at the end of the year and to be at increased odds of not being retained into the second year of study.

\section{Method}

\subsection{Participants and procedure}

A newsletter including the link to the on-line emotion management test and the various questionnaires was sent to all new first year students at a university in the North West of England, UK. Students were also recruited directly in core lectures for each discipline across the university. The university is a mid-sized institution with approximately 20,000 undergraduates who study subjects as diverse as Astrophysics, Art and Design, Dentistry, Fashion, and Nursing. A total of 509 (51\% male; 91\% categorized themselves as White/British) participants completed the on-line measures during the first 3 weeks of their first year at university and gave active consent to obtain individual data linked to entry qualifications, grade point average at the end of year one, and retention data from the university system. $65 \%(\mathrm{~N}=331)$ of these participants were retained at a further two time points (3 and 6 months after starting their first year). To minimize the bias associated with this attrition, we used the expectation maximization (EM) algorithm to impute any missing data for these remaining 331 participants (Schafer \& Graham, 2002). This algorithm assumes the data are missing completely at random (Little \& Rubin, 1987; Schafer \& Graham, 2002). Of the 331 students (those with data for at least two of the three measurement waves), 3\% of data missing were missing. Participants with and without complete data were compared using Little’s (1988) Missing Completely At Random (MCAR) test. This yielded a non-significant chi-square value $\left(\chi^{2}=54.36, n s\right)$, suggesting that missing values could be reliably estimated. 
Thus, all analyses were conducted on the sample of 331 participants. Table 1 provides demographic information regarding the sample.

To ensure that this final sample of 331 participants was no different to the original sample of 509 participants on predictor or adjustment variables, we conducted ANOVAs comparing retained and drop-out groups on the variables in this study. Analyses showed participants retained were no different to those that had dropped out at that particular time point on all predictor and adjustment variables $\left(F \geq 1.27 p \geq .287, \eta \mathrm{p}^{2} \leq .024\right)$, and on GPA at the end of the year $\left(F \geq .939 p \geq .392, \eta p^{2} \leq .006\right)$. Chi-square analyses also showed that those who dropped out of the study were no more likely to fail their course than students who were retained $\left(\chi^{2} \geq 2.450 \mathrm{p} \geq .654\right)$.

\subsection{Measures}

\subsubsection{University adjustment}

The College Adaptation Questionnaire (CAQ; Crombag, 1968; Vlaander \& van Rooijen, 1981) measured adjustment to university. 18 statements, scored on a 7-point scale, measure individuals’ psychological, social and interpersonal adaptation to university. Ten of the items reflect poor adjustment (e.g. “I find it hard to get used to life here”); eight items reflect positive adjustment (e.g. 'I am glad that I came to study here’). The score for the CAQ is the sum of the item scores after reverse coding the 10 'poor adjustment' items; high scores on the CAQ represent higher adjustment to university. The CAQ was reliable at each time point: alpha was $0.88,0.85$, and 0.82 , comparable to previous research $(\alpha=0.83$ : van Rooijen, 1986).

To validate the latent classes, we examined differences over time on two further measures of psychological adjustment (loneliness and depression) between people following the different developmental trajectories of adjustment. Loneliness and depression data were collected at all three time- points. Loneliness was measured using the UCLA Loneliness 
Scale Revised (Russell, 1996). Depression was measured using the CES-D Depression Scale (Radloff, 1977). The CES-D scale is designed to measure depressive symptoms in the general population. Higher scores on these measures indicate greater loneliness and depression respectively. Reliability was good for loneliness $(\alpha=.91, .89$, and .92 at Time 1,2 , and 3 respectively) and depression ( $\alpha=.75, .85$, and .82 at Time 1,2 , and 3 respectively).

\subsubsection{Predictors of university adjustment}

The following items were measured at T1 only.

Demographics. Students provided information regarding their gender, age, term-time residence, part-time or full-time study, home or international student, first language, ethnicity, and disability. Information related to students’ previous qualifications upon entry into university was extracted from the university system: within the UK, these are known as UCAS point scores.

AEI: Emotion Management. The Situational Test of Emotion Management (STEM: MacCann \& Roberts, 2008) assessed emotion management skills. In the STEM, participants are presented with details of 30 emotional situations; for each one, participants select the most effective course of action from four possible options. The participant's choice is assigned a numerical value based on expert ratings. So, if the average expert score for Option B for situation 22 was 4.6, participants selecting Option B for statement 22 would score 4.6. Thus, scores reflect a person's actual understanding of correct emotion management strategies in given situations according to expert opinion. Higher scores suggest better understanding of emotion management. Internal consistency of the STEM is good $\alpha=0.92$ (MacCann $\&$ Roberts, 2008).

TEI: Emotional Self-efficacy. The Emotional Self-Efficacy Scale (ESES: Kirk, Schutte \& Hine, 2008) comprises 32 items assessing self-perception of emotion-related skills. Participants rate their responses using a 5-point Likert scale ranging from "not at all 
confident” to "very confident”. Four factors have been proposed for the ESES (Dacre-Pool \& Qualter, 2012a): (1) using and managing your own emotions, (2) identifying and understanding your own emotions, (3) dealing with emotions in others, and (4) perceiving emotion through facial expressions and body language. In the current study, reliability of the ESES subscales was good ( $\alpha=0.79$ [Factor 4$]-.91$ [Factor 1$])$.

\subsubsection{Outcome measures}

Academic Achievement and Retention Data. Students’ Grade Point Average (GPA) was obtained from the university system at the end of the first year. Details of which students had been retained into the second year of study were extracted from the university's central database.

\subsection{Overview of data analyses}

First, we used latent growth curve modeling (LGCM) in Mplus 5.1 (Muthén \& Muthén, 1998-2008) to estimate both the initial level of adjustment at baseline (intercept) and the rate of change in adjustment from baseline across time (slope) for the whole sample (Duncan, Duncan, \& Strycker, 2006). LGCM provides a picture of how the chosen construct (CAQ adjustment score) changes for the full sample over time. Model fit was assessed using

global fit indices: $\chi^{2}$ (should be a low as possible), CFI (with a cut-off value of .95) and RMSEA (should be between .05 and .08 for a reasonable fit to the data) (Hu \& Bentler, 1999). The variance in the estimates was also examined, which would justify an examination of inter-individual differences in the trajectory of adjustment over time.

Because there was significant variance for the estimates of intercept and slope, we employed latent growth mixture modeling (LGMM) to identify discrete growth classes (groups) of university adjustment (Muthén \& Shedden, 1999). We validated these latent classes by determining whether they were also different over time on loneliness and depression. 
The LGMM ${ }^{1}$ was conducted in Mplus version 5.1. Within the model, within-class variances were freely estimated instead of fixed to zero. A series of models, with varying numbers of classes, were fit to the CAQ data to determine (a) the optimal number of latent classes that underlie the university adjustment data, and (b) the form of changes over time in adjustment within each class. We used several criteria in guiding our decision about number of latent classes: one set of criteria had to do with the substantive meaning and theoretical conformity of the extracted classes (Muthén, 2003), which was determined in part by whether they were predicted by and predicted other variables in the model well (see Steps 2 and 3 of the LGMM). We also used a number of statistical tests and indices to help in this decision process (McLachlan\& Peel, 2000): The Akaike Information Criteria (AIC), Bayesian information criteria (BIC), Adjusted BIC, Entropy, and the Lo-Mendell-Rubin likelihood ratio test (LMR).

In the second stage of the LGMM, we added covariates to the model and explored whether information collected at the start of the academic year predicted membership of the adjustment classes. All variables were centered before running the model to avoid multicollinearity. In this stage of the LGMM, the classes were simultaneously modeled as latent nominal outcome variables using logistic regression.

In the third stage of the LGMM, we examined the relation between adjustment classes and academic outcome by adding these variables to the model. Within Mplus, the relation between the outcome variables and the trajectories within the full model was examined using the Wald chi-square test, which tests the equality of outcome means across latent classes.

\section{Results}

\subsection{Identifying different classes of first year CAQ scores}

LGCM results showed the intercept was significant, but the slope was not $\left(\beta_{0}\right.$ (intercept) $=$ 88.98, $\mathrm{p}<.001, \beta_{1}$ (slope) $=-.08, \mathrm{p}=-.207$ ), indicating that participants, on average, scored 
88.98 on the CAQ at Time 1 and this level of adjustment was stable over time $\left(\chi^{2}[\mathrm{df}=3]\right.$, = 12.320, CFI $\geq .976, T L I \geq .953$, RMSEA $=.078)$. However, the estimates of variance related to the intercept and the slope were significant $(\mathrm{p}<.005)$, which justified an examination of interindividual differences in adjustment scores over time using LGMM.

Using LGMM, four latent classes of university adjustment were identified. Table 2 summarizes the fit statistics for the 1-4 models. We stopped at 4 classes because no more classes with a prevalence of at least $1 \%$ could be extracted. Figure 1 presents the four-class solution, which includes a group of students whose reported adjustment remained low over the course of the study (low, stable adjustment, $n=102,31 \%$ of sample), a group who reported average levels of adjustment over time (medium, stable adjustment, $n=128,39 \%$ of sample), and a group whose adjustment levels were very high and stable over the six months (high, stable adjustment, $\mathrm{n}=73,22 \%$ of sample). The final group (low, increasing adjustment, $\mathrm{n}=28,8 \%$ of sample), included students whose adjustment was low at the start of the year, but increased to above average levels by Time 3. Intercept and slope estimates and descriptive statistics for adjustment by time point and latent class are presented in Table 3.

To validate the adjustment groups, we examined differences on depressive symptoms and loneliness between the groups. First, we found that at each time point, higher scores on the CAQ were significantly correlated with loneliness ( $r s>.23$, ps $>.001)$ and depressive symptoms (rs $>.26$, $p$ s $>.001)$. Results showed that the adjustment groups differed on loneliness at each time point $(F \mathrm{~s}>3.70, p s<.01)$ and on their self-reported depressive symptoms (Fs $>24.8, p s<.001)$. Means are shown in Table 3. Post-hoc tests showed that students in the high, stable adjustment group reported the lowest levels of loneliness and depressive symptoms; students in the low, stable adjustment group reported the highest levels of loneliness and depressive symptoms. In the low increasing adjustment group, we saw an 
accompanying reduction in loneliness and depression, although these remained higher than scores for the medium and high adjustment groups.

\subsection{Predictors of adjustment trajectory class}

Results examining predictors showed that the low, stable adjustment class could be differentiated from the average and high adjustment groups (Table 4). Students who had lower emotion management scores and lower ESE scores were at increased odds of belonging to the low, stable adjustment group; membership of the high stable adjustment group was determined by high scores on the emotional management test and ESE. Further, those following a low, but increasing adjustment trajectory were different to the low, stable adjustment group in terms of having higher emotion management skills and higher ESE for identifying and understanding their own emotions and in dealing with emotions in others. This low, increasing group was also different to all other trajectory groups: having higher ESE factor 3 scores (able to deal with emotions in others) increased the odds of belonging to the low, increasing trajectory group.

STEM and emotional-self efficacy scores were not highly or significantly correlated $\left(r_{\mathrm{s}} \geq 0.007\right.$ and $\left.\leq .145, \mathrm{ps}>.05\right)$, suggesting that having the skills to manage emotions and the confidence to deal with emotional information are different. Taken together, the findings suggest that whilst AEI and TEI are independent constructs, they, somehow, work together to predict adjustment outcome.

No demographics other than age predicted membership of the adjustment groups. For age, being older at Time 1 predicted membership of the high, stable adjustment group and the low, increasing adjustment group.

\subsection{Academic outcomes as a function of first year adjustment class}

We added academic achievement as an outcome measure into our model to examine whether adjustment class predicted academic achievement. Wald’s tests of mean equality, 
showed that being a member of the low stable adjustment group predicted low academic performance even when entry qualifications were controlled $\left(\chi^{2}=26.64 \mathrm{p}<0.001\right)$. Descriptive statistics on academic achievement by adjustment latent class are summarized in Table 5.

It was impossible to examine whether membership of a particular adjustment group predicted retention because all students who were not retained in the current study dropped out of university within the first three months and provided adjustment data only at Time 1 . Examination of whether scores on the CAQ at Time 1 predicted withdrawal from the course was examined using a Wald's Chi-square test of significance in SPSS. Analysis showed poor adjustment scores at Time 1 predicted withdrawal from the course $\left(\chi^{2}=3.89 \mathrm{p}=0.043\right)$.

\section{Discussion}

This study is the first to prospectively describe first year adjustment to university in the UK. The study investigated whether (1) students followed different developmental trajectories of adjustment, (2) AEI and TEI, specifically emotion management and emotional self-efficacy, independently predicted membership of these adjustment groups, and (3) students following particular trajectories of adjustment were more at risk of withdrawal from university, and academic underachievement.

The results indicated that adjustment was heterogeneous, supporting the earlier findings of Duchesne et al. (2007). Because growth mixture modeling is a sample-specific technique, it is not surprising that we found different trajectory groups compared to Duchesne et al. In support of our aims, we identified four distinct adjustment groups: (1) low, stable adjustment, (2) medium, stable adjustment, (3) high, stable adjustment, and (4) low, increasing adjustment. It is of some concern that 31\% showed low, stable adjustment, because this group was characterized by the lowest scores on the CAQ, the highest scores on the depression and loneliness measures, and the worst academic performance. We also found 
evidence that aspects of AEI and TEI are important in the successful implementation of coping strategies linked to university adjustment. For example, low emotion management scores and low emotional self-efficacy predicted membership of the low, stable adjustment group; higher skills of emotion management and emotional self-efficacy predicted membership of the high, stable adjustment group. Emotional self-efficacy and emotion management also predicted increased adjustment for some students who had initial poor adjustment to university. These results suggest that emotion management skills and emotional self-efficacy are important for successful adjusting to university life.

Previous research shows that having higher AEI and TEI has direct positive effects on academic performance amongst undergraduates (Austin et al., 2005; Lyons \& Schneider, 2005; Parker et al., 2004). Our findings extend research by showing that specific aspects of AEI and TEI, namely emotion management and emotional self-efficacy, impact academic performance indirectly through adjustment.

Our findings also support recent empirical work where emotion management was shown to influence students’ adaptation to specific and general university stressors (Tariq et al., 2012; MacCann et al., 2011). We advance that literature by showing emotional self-efficacy, too, predicts adaptation to university stressors to impact academic success; even though emotional self-efficacy was not correlated with emotion management, it appeared to offer protection against stable low adjustment. This finding offers further support to the notion that both AEI and TEI promote positive ways of coping with stressful situations (Davis \& Humphrey, 2012), which leads to adaptation (Keefer, et al. 2009, 2012). Future research should examine how emotion management skills and self-efficacy provide such protection.

Our results showed that adjustment and academic achievement did not follow a simple linear path, with the medium, stable adjustment group having a higher GPA than the high, stable adjustment group when entry qualification were controlled. Also, by the end of $1^{\text {st }}$ year 
the low, increasing group showed similar levels of adjustment to the high, stable group, yet surpassed this latter group academically. Results seem to indicate that the medium, stable and low, increasing adjustment groups share similar traits. There was no significant difference in emotion management scores between these two groups, and the only significant difference in emotional self-efficacy scores was for ESES factor 3 (dealing with emotions in others). These two groups also achieved the highest GPA, with little difference between grades. It is not clear why these two groups had the most academic success, and further research is needed to answer this question.

\subsection{Implications of findings}

Our results have implications for student well-being interventions. Findings suggest students who appear to have poor adjustment to university should not be treated as a homogeneous group when planning interventions, as their needs may be quite different. The low, increasing adjustment group had higher emotion management and emotional selfefficacy and seemed to adjust quickly to university life, despite poor initial adjustment. The higher scores on emotion management and emotional self-efficacy suggest that this group has the skills required to recognize their emotions and cope with stressors; they are able and confident to access support if it is available to them. Thus, having services clearly marked for these students will be useful, e.g. signposting social support networks.

Universities might offer different interventions for members of the low, stable adjustment group, who have poor emotion management and low levels of emotion selfefficacy. Universities could identify students with these low scores at the beginning of the year and intervene to increase their emotion management skills and emotional self-efficacy (Dacre-Pool \& Qualter, 2012b). Such interventions increase the likelihood of students developing adaptive coping strategies, seeking support, and being able to stay calm under pressure, which may lead to better adjustment and academic success. The benefits for 
university adjustment and academic achievement of using an EI intervention in HE have been demonstrated elsewhere (Dacre-Pool \& Qualter, 2012b; Nelis, Quoidbach, Mikolajczak, \& Hansenne, 2009; Qualter et al., 2009).

\subsection{Limitations and future directions}

One limitation of the present study is that academic achievement was only assessed for a single academic year. Further examination of the complex interplay between emotion management, emotional self-efficacy, adjustment and academic success over a longer period of time is needed to determine whether the effects noted in the current study carry over to subsequent years. Based on findings from Keefer et al. (2012), we might expect the effects of emotion management and emotional self-efficacy on future academic performance and final graduation outcome to be less robust than those for first-year retention rates or for university adjustment in year 1 .

There are other limitations of the current study that can be improved in future empirical work. For example, it would be useful to differentiate between reasons for withdrawal from university. So, has the student gained confidence to transfer to a different course (which may be seen as a positive action), or have they dropped out entirely (which may be more negative). Also, personality and ability were not controlled in the current analyses; whilst we included a proxy measure of intelligence in the form of student entry grades, future research should examine the unique effects of emotion management and emotional self-efficacy independent of intelligence and personality. Future research would also benefit from the inclusion of a more diverse range of ethnic backgrounds to examine if there are any cultural differences in adjustment to university, a larger sample to allow the exploration of other trajectories, and more time points to allow examination of cubic and quadratic growth.

\subsection{Conclusions}


Running Head: Trajectories of first year university adjustment in the UK 19

Our findings provide new insights regarding university adjustment, its predictors, and how it impacts on academic success. We found that poor, stable adjustment was predicted by low levels of both emotion management and emotional self-efficacy, which then predicted lower academic achievement, even when controlling for entry qualifications. We also found that high emotion management and emotional self-efficacy protected against initial low levels of adjustment. These findings have important implications for intervention programs. 
Running Head: Trajectories of first year university adjustment in the UK 20

\section{Footnotes}

1. Please note that Structural Equation Modeling and regression analyses are not appropriate for the current study because they aim to predict outcome by examining the association between independent and dependent variables; LGMM is a person-centered approach that aims to sort individuals into groups of individuals who are similar to each other and different from those in other groups, and then estimate varying class membership probabilities as a function of covariates (predictors) and predict outcomes of the latent class variable (Duncan, Duncan, \& Strycker, 2006; Marsh, Lüdtke, Trautwein, \& Morin, 2009). 


\section{References}

Austin, E. J., Evans, P., Goldwater, R., \& Potter, V. (2005). A preliminary study of emotional intelligence, empathy and exam performance in first year medical students. Personality and Individual Differences, 39, 1395-1405.

Austin, E. J., Saklofske, D. H., \& Mastoras, S. M. (2010). Emotional intelligence, coping, and exam-related stress in Canadian undergraduate students. Australian Journal of Psychology, 62, 42-50.

Baker, S. R. (2004). Intrinsic, extrinsic, and motivational orientations: Their role in university adjustment, stress, well-being, and subsequent academic performance. Current Psychology, 23 (3), 189-202.

Bandura, A. (1986). Social Foundation of thought and action. New Jersey: Prentice Hall.

Beyers, W., \& Goossens, L. (2002). Concurrent and predictive validity of the Student Adaptation to College Questionnaire in a sample of European freshman students. Educational and Psychological Measurement, 62, 527-538.

Brackett, M., Rivers, S. E., \& Salovey, P. (2011). Emotional intelligence: implications for personal, social, academic, and workplace success. Social and Personality Psychology Compass, 5(1), 88-103.

Christie, H., Munro, M., \& Fisher, T. (2004). Leaving university early: Exploring the differences between continuing and non-continuing students. Studies in Higher Education, 29, 617-636.

Cooke, R., Bewick, B. M., Barkham, M., Bradley, M., \& Audin, K. (2006). Measuring, monitoring and managing the psychological well-being of first year university students. British Journal of Guidance \& Counselling, 34 (4), 505-517. 
Crombag, H. F. M. (1968). Studiemotivatie en Studieattitude. Groningen, Wolters.

Dacre-Pool, L., \& Qualter, P. (2012a). The dimensional structure of the emotional selfefficacy scale (ESES). Australian Journal of Psychology, 64, 147-154

Dacre-Pool, L.* \& Qualter, P. (2012b). Developing Emotional Intelligence and Emotional Self-Efficacy: The effect of a teaching intervention for university students. Learning and Individual Differences, 22, 306-312

Dahmus, S., Bernardin, H. J., \& Bernardin, K. (1992). Test review: Student adaptation to college questionnaire. Measurement and Evaluation in Counseling and Development, 25, 139-142.

Davis, S. K., \& Humphrey, N. (2012). The influence of emotional intelligence (EI) on coping and mental health in adolescence: Divergent roles for trait and ability EI. Journal of Adolescence, 35, 1369-1379.

Duchesne, S., Ratelle, C. F., Larose, S., \& Guay, F. (2007). Adjustment trajectories in college science programs: Perceptions of qualities of parents’ and college teachers’ relationships. Journal of Counseling Psychology, 54 (1), 62-71.

Duncan, T.E., Duncan, S.C., \& Strycker, L.A. (2006). An introduction to latent variable growth curve modeling ( $2^{\text {nd }}$ Edition). New Jersey: Lawrence Erlbaum Associates Inc.

Engelberg, E., \& Sjoberg, L. (2004). Emotional Intelligence, affect intensity, and social adjustment. Personality and Individual Differences, 37, 533-542.

Galatzer-Levy, I. R., Burton, C. L., \& Bonanno, G. A. (2012). Coping flexibility, potentially traumatic life events, and resilience: A prospective study of college student adjustment. Journal of Social and Clinical Psychology, 31, 542-567. 
Gall, T. L., Evans, D. R., \& Bellerose, S. (2000). Transition to first-year university: Patterns of change in adjustment across life domains and time. Journal of Social and Clinical Psychology, 19 (4), 544-567.

Gerdes, H., \& Mallinckrodt, B. (1994). Emotional, social, and academic adjustment of college students: A longitudinal study of student retention. Journal of Counseling \& Development, 72, 281-288.

Hu, L., \& Bentler, P. M. (1999). Cutoff criteria for fit indexes in covariance structure analysis: Conventional versus new alternatives. Structural Equation Modeling, 6, 1-55.

Keefer, K. V., Parker, J. D. A., \& Saklofske, D. H. (2009). Emotional intelligence and physical health. In C. Stough, D. H. Saklofske, \& J. D. A. Parker (Eds.), The Springer series on human exceptionality. Assessing emotional intelligence: Theory, research, and applications (pp. 191-218).

Keefer, K.V., Parker, J.D.A., \& Wood, L.M. (2012). Trait Emotional Intelligence and University Graduation Outcomes: Using Latent Profile Analysis to Identify Students at Risk for Degree Noncompletion. Journal of Psychoeducational Assessment, 30, 402413

Kirk, B. A., Schutte, N. S., \& Hine, D. W. (2008). Development and preliminary validation of an emotional self-efficacy scale. Personality and Individual Differences, 45, 432436.

Little, R. J. A. (1988). A test of missing completely at random for multivariate data with missing values. Journal of the American Statistical Association, 83 (404), 1198-1202.

Little, R. J. A., \& Rubin, D. B. (1987). Statistical analysis with missing data. New York: John Wiley. 
Lyons, J. B., \& Schneider, T. R. (2005). The influence of emotional intelligence on performance. Personality and Individual Differences, 39, 693-703.

MacCann, C., Fogarty, G. J., Zeidner, M., \& Roberts, R. D. (2011). Coping mediates the relationship between emotional intelligence (EI) and academic achievement. Contemporary Educational Psychology, 36 (1), 60-70.

MacCann, C., \& Roberts, R. D. (2008). New paradigms for assessing emotional intelligence: Theory and data. Emotion, 8 (4), 540-551.

Marsh H., Lüdtke O., Trautwein U., \& Morin A. (2009) Classical latent profile analysis of academic self-concept dimensions: synergy of person- and variable-centered approaches to theoretical models of self-concept. Structural Equation Modeling, 16, 191-225.

Mayer, J. D., Salovey, P., \& Caruso, D. (2004). Emotional intelligence: Theory, findings, and implications. Psychological Inquiry, 15, 197-215.

McLachlan, G. J., \& Peel, D. (2000). Finite mixture models. New York: Wiley.

Muthén, B. (2003). Statistical and substantive checking in growth mixture modeling: Comment on Bauer and Curran (2003). Psychological Methods, 8, 369-377.

Muthén, B. (2004). Latent variable analysis: Growth mixture modeling and related techniques for longitudinal data. In Kaplan, D. Handbook of quantitative methodology for the social sciences. Newbury Park, CA: Sage Publications, 345-368.

Muthén, B., \& Shedden, K. (1999). Finite mixture modeling with mixture outcomes using the EM algorithm. Biometrics, 55, 463-469.

Muthén, L.K., \& Muthén, B. (1998-2008). Mplus users guide (5). Los Angeles: Author.

Nagin, D. S. (1999). Analyzing developmental trajectories: a semiparametric, group-based 
approach. Psychological Methods, 4, 139-157.

Nelis, D., Quoidbach, J., Mikolajczak, M., \& Hansenne, M. (2009). Increasing emotional intelligence: (How) is it possible? Personality and Individual Differences, 47, 36-41.

Parker, J. D. A., Hogan, M. J., Eastabrook, J. M., Oke, A., \& Wood, L. M. (2006). Emotional intelligence and student retention: Predicting the successful transition from high school to university. Personality and Individual Differences, 41, 1329-36.

Parker, J. D. A., Summerfeldt, L. J., Hogan, M. J., \& Majeski, S. A. (2004). Emotional intelligence and academic success: Examining the transition from high school to university. Personality and Individual Differences, 36, 163-72.

Petrides, K. V., Furnham, A., \& Mavroveli, S. (2007). Trait emotional intelligence: Moving forward in the field of EI. In G. Matthews, M. Zeidner, \& R. Roberts (Eds.), Emotional intelligence: Knowns and unknowns (Series in Affective Science). Oxford: Oxford University Press.

Petrides, K., Pita, R., \& Kokkinaki, F. (2007). The location of trait emotional intelligence in personality factor space. British Journal of Psychology, 98, 273-289.

Prillerman, S. L., Myers, H. F., \& Smedley, B. D. (1989). Stress, well-being, and academic achievement in college. In G.L.V. Berry \& J.K. Asamen (Eds.), Black students: Psychosocial issues and academic achievement (pp. 198-217). Thousand Oaks, CA: Sage.

Pritchard, M. E., Wilson, G. S., \& Yamnitz, B. (2007). What predicts adjustment among college students? A longitudinal panel study. Journal of American College Health, 56, $15-21$. 
Qualter, P., Whiteley, H., Morley, A., \& Dudiak, H. (2009). The role of emotional intelligence in the decision to persist with academic studies in HE. Research in PostCompulsory Education, 14 (3), 219-231.

Radloff, L. S. (1977). The CES-D scale: A self-report depression scale for research in the general population. Applied Psychological Measurement, 1, 385-401.

Rickinson, B., \& Rutherford, D. (1995). Increasing undergraduate student retention rates. British Journal of Guidance \& Counselling, 23 (2), 161-172.

Russell, D. (1996). The UCLA Loneliness Scale (version 3): Reliability, validity, and factor structure. Journal of Personality Assessment, 66, 20-40.

Saklofske, D. H., Austin, E. J., Galloway, J., \& Davidson, K. (2007). Individual difference correlates of health-related behaviors: Preliminary evidence for links between emotional intelligence and coping. Personality and Individual Differences, 42, 491-502.

Schafer, J. L., \& Graham, J. W. (2002). Missing data: Our view of the state of the art. Psychological Methods, 7 (2), 147-177.

Sennett, J., Finchilescu, G., Gibson, K., \& Strauss, R. (2003). Adjustment of black students at a historically white South African university. Educational Psychology, 23, 107-116.

Soucy, N., \& Larose, S. (2000). Attachment and control in family and mentoring contexts as determinants of adolescent adjustment to college. Journal of Family Psychology, 14, 125-143.

Strahan, E. Y. (2003). The effects of social anxiety and social skills on academic performance. Personality and Individual Differences, 34, 347-366.

Swanson, V., Broadbridge, A. and Karatzias, A. (2006), Earning and learning: Role 
congruence, state/trait factors and adjustment to university life. British Journal of Educational Psychology, 76: 895-914.

Tariq, V. N., Qualter, P., Roberts, S., Appleby, Y. and Barnes, L. (2013) Mathematical literacy in undergraduates: role of gender and emotional intelligence. International Journal of Mathematical Education in Science and Technology. i-First, doi:10.1080/0020739X.2013.770087

Tao, S., Dong, Q., Pratt, M. W., Hunsberger, B., \& Pancer, S. M. (2000). Social support: Relations to coping and adjustment during the transition to university in the People's Republic of China. Journal of Adolescent Research, 15 (1), 123-144.

van Roojen, L. (1986). Advanced students' adaptation to college. Higher Education, 15 (3/4), 197-209.

Vlaander, G. P. J., \& van Roojen, L. (1981). Nieuwe gegevens over de Aanpassingsvragenlijst (New data about the adjustment questionnaire). Tijdschrift voor Onderwijsresearch, 6, 33-37.

Weko, T. (2004). Old Dogs and New Tricks: What Can the UK Teach the US about Higher Education? London: Higher Education Policy Institute. 
Table 1. Characteristics of the sample.

\section{Demographics at T1}

Age in years at T1 (mean)

20.32

Age range in years

$18-49$

Male \%

46.37

Entrance Qualifications (mean UCAS points)

310.64

UCAS points - range

$120-560$

Holds English GCSE or above \%

62.57

Holds Maths GCSE or above \%

63.69

White British \%

87.15

Living in Halls of residence \%

48.60

International students \%

English as first language \%

92.18

Declared disability \%

15.08

Full-time student \%

98.32 
Table 2. Conditional latent class analysis for first year university adjustment: Fit statistics.

\begin{tabular}{llllll}
\hline Class & AIC & BIC & Adj_BIC & Entropy & LRT $p$ value \\
(Group) & & & & \\
\hline 1 & 7274.96 & 7305.43 & 7280.05 & - & \\
2 & 7209.57 & 7209.57 & 7165.16 & .97 & .301 \\
3 & 7156.36 & 7221.10 & 7167.18 & .95 & .183 \\
$\mathbf{4}$ & $\mathbf{7 1 2 3 . 0 1}$ & $\mathbf{7 2 0 0 . 1 5}$ & $\mathbf{7 1 3 6 . 7 1}$ & $\mathbf{. 9 5}$ & $<.001$ \\
\hline
\end{tabular}

Notes: $\mathrm{AIC}=$ Akaike Information Criteria $; \mathrm{BIC}=$ Bayesian information criteria; Adj BIC = adjusted BIC; LRT = Lo-Mendell-Rubin test. Lower AIC, BIC, and adjusted BIC values indicate a more parsimonious model. Entropy is a measure of classification accuracy, with values closer to 1 indexing greater precision (range: 0 -1); the LMR test provides a $k-1$ likelihood ratio-based method for determining the ideal number of trajectories, with low $p$ value indicating a better fit to the data. We stopped at 4 classes because no more classes with a prevalence of at least $1 \%$ could be extracted. BOLD = those goodness of fit indexes with best results. The fit indexes reported here are from the model with all covariates (both predictors and outcome) included. The class membership changed slightly when the covariates were added to the model, but, for reasons of parsimony, the changes in class membership and fit indexes are not noted here; they are available from the corresponding author upon request. 
Figure 1. Four-class solution for first year university adjustment.

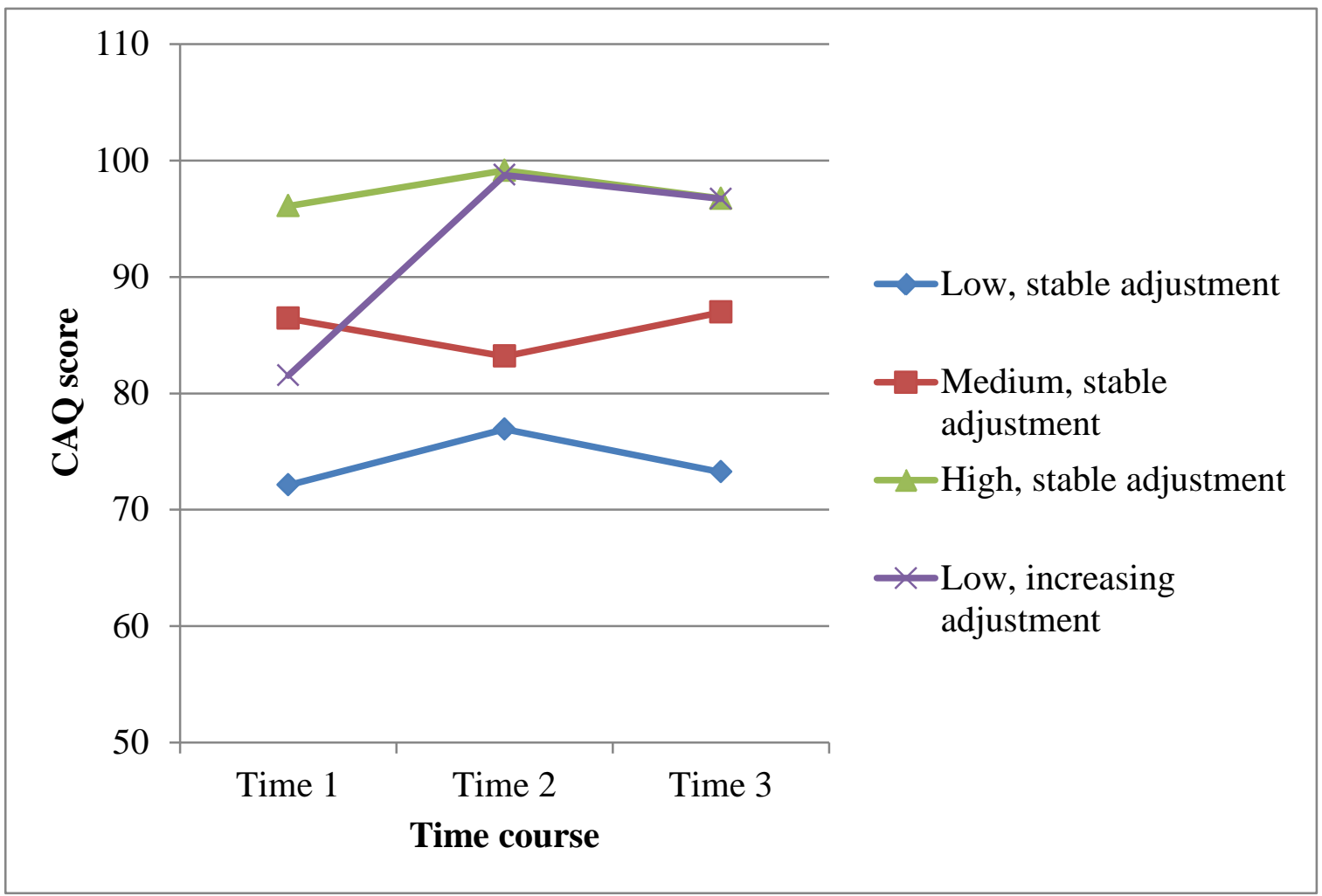

Notes: Time 1 = three weeks after initial entry into university; Time $2=3$ months after entry into Year 1; Time 3 = 6 months after entry into Year 1. Low, stable adjustment $(\mathrm{N}=102)$; medium, stable adjustment $(\mathrm{N}=128)$; high adjustment $(\mathrm{N}=73)$; increasing adjustment $(\mathrm{N}=$ 28). Scores are adjusted for age at entry into university, emotional self-efficacy score, and emotion management score that were shown to predict group membership in LGMM. 
Table 3. Intercept and slope estimates for each trajectory and university adjustment, loneliness and depression scores (and standard deviation) over time by adjustment group.

\section{University Adjustment Group}

\begin{tabular}{|c|c|c|c|c|c|}
\hline & Low, stable & $\begin{array}{l}\text { Medium, } \\
\text { stable }\end{array}$ & High, stable & $\begin{array}{c}\text { Low, } \\
\text { increasing }\end{array}$ & $\begin{array}{l}\text { Whole } \\
\text { sample }\end{array}$ \\
\hline $\mathbf{N}=$ & 102 & 128 & 73 & 28 & 331 \\
\hline \multicolumn{6}{|l|}{ LGMM } \\
\hline Intercept & $72.02 *$ & $86.71^{*}$ & $95.30 *$ & $82.12 *$ & .88 \\
\hline Slope & .09 & -.22 & -.08 & $2.48 *$ & -.08 \\
\hline \multicolumn{6}{|l|}{ CAQ } \\
\hline Time 1 & 72.12 (6.15) & 86.44 (5.96) & 96.09 (4.75) & $81.53(5.20)$ & 85.96(10.32) \\
\hline Time 2 & 76.92 (8.30) & 83.18 (4.95) & 99.14 (6.69) & $98.76(4.75)$ & 92.17 (9.55) \\
\hline Time 3 & 73.24 (5.99) & 86.97 (5.54) & 96.73 (4.73) & $96.71(5.25)$ & 96.34 (9.64) \\
\hline \multicolumn{6}{|c|}{ Loneliness } \\
\hline Time 1 & $51.56(9.50)$ & 39.44 (5.98) & 36.38 (8.46) & 44.28 (8.67) & 42.35(9.89) \\
\hline Time 2 & 51.54 (8.52) & 38.62 (7.87) & 34.97 (8.52) & $42.06(9.25)$ & $41.50(10.35)$ \\
\hline Time 3 & $50.52(7.53)$ & $38.95(10.14)$ & 32.53 (7.95) & 39.50 (9.38) & 39.84(10.84) \\
\hline
\end{tabular}

\section{Depression}

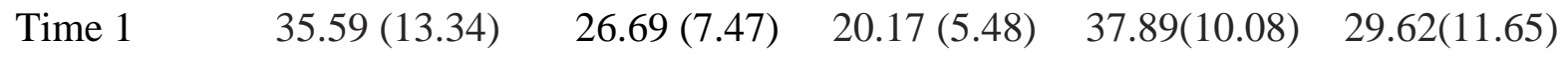

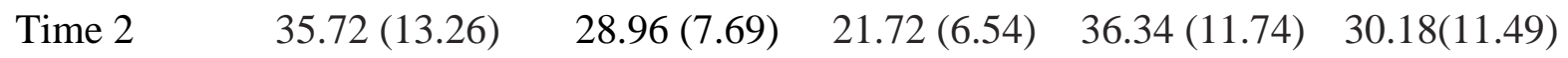

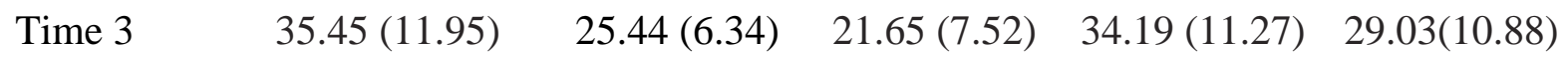

Notes: LGMM = Latent Growth Mixture Modeling; intercept = initial level of adjustment at baseline (intercept); slope $=$ rate of change in adjustment from baseline across time; * $=p$ $<.001$; Time 1 = three weeks after initial entry into university; Time $2=3$ months after entry 
Running Head: Trajectories of first year university adjustment in a UK population 32

into Year 1; Time $3=6$ months after entry into Year 1. Scores for CAQ are adjusted for missing data via EM (expectation maximization) algorithm-adjusted means and covariates. Scores are also adjusted for age at entry into university. 
Table 4. Latent growth mixture modeling (LGMM) of university adjustment predicted by demographic and individual predictors.

\begin{tabular}{|c|c|c|c|c|c|c|c|c|c|c|c|c|}
\hline & \multicolumn{12}{|c|}{ University adjustment class comparison } \\
\hline & \multicolumn{2}{|c|}{ Medium, stable } & \multicolumn{2}{|c|}{ High, stable } & \multicolumn{2}{|c|}{ Low, increasing } & \multicolumn{2}{|c|}{ High, stable } & \multicolumn{2}{|c|}{ High, stable } & \multicolumn{2}{|c|}{ Medium, stable } \\
\hline & \multicolumn{2}{|c|}{ adjustment vs. low, } & \multicolumn{2}{|c|}{ adjustment vs. low, } & \multicolumn{2}{|c|}{ adjustment vs. low, } & \multicolumn{2}{|c|}{ adjustment vs. } & \multicolumn{2}{|c|}{ adjustment vs. } & \multicolumn{2}{|c|}{ adjustment vs. } \\
\hline & \multicolumn{2}{|c|}{ stable adjustment } & \multicolumn{2}{|c|}{ stable adjustment } & \multicolumn{2}{|c|}{ stable adjustment } & \multicolumn{2}{|c|}{ medium, stable } & \multicolumn{2}{|c|}{ low, increasing } & \multicolumn{2}{|c|}{ low, increasing } \\
\hline & & & & & & & \multicolumn{2}{|c|}{ adjustment } & \multicolumn{2}{|c|}{ adjustment } & \multicolumn{2}{|c|}{ adjustment } \\
\hline Variable & OR & $p$ value & OR & $p$ value & OR & $p$ value & OR & $p$ value & OR & $p$ value & OR & $p$ value \\
\hline Age† & 0.99 & .310 & 1.19 & $.003 * *$ & 1.13 & $.024 *$ & 1.03 & .696 & 0.96 & .346 & 0.94 & .329 \\
\hline STEM & 1.15 & $0.043^{*}$ & 1.27 & $0.006 * *$ & 1.18 & $0.019 * *$ & 1.22 & $0.009 * *$ & 1.24 & $0.008 *$ & 1.00 & 0.975 \\
\hline ESES factor 1 & 1.12 & $0.041^{*}$ & 1.21 & $0.008 * *$ & 0.99 & 0.439 & 1.03 & 0.569 & 1.08 & 0.420 & 0.96 & 0.620 \\
\hline ESES factor 2 & 1.16 & $0.036^{*}$ & 1.39 & $0.035 *$ & 1.72 & $<0.001^{* * *}$ & 1.06 & 0.490 & 1.03 & 0.173 & 0.86 & 0.292 \\
\hline ESES factor 3 & 1.18 & $<0.02 * *$ & 1.38 & $0.008^{* *}$ & 1.29 & $0.032 *$ & 1.13 & $0.013^{*}$ & 1.38 & $0.009 * *$ & 1.34 & $0.013 *$ \\
\hline ESES factor 4 & 1.00 & 0.730 & 1.79 & $<.001 * * *$ & 1.02 & 0.394 & 1.29 & $<0.001^{* * *}$ & 1.28 & $0.014^{*}$ & 0.98 & 0.936 \\
\hline
\end{tabular}

Notes. OR = odds ratio. $†$ The only significant demographic predictor was age; the other factors did not predict group membership and so are not included in this table. ${ }^{*} p<0.05 ;{ }^{* *} p<0.01 ;{ }^{* * *} p<0.001 .4$ factors of the ESE are as follows: factor $1=$ using and managing your own emotions, factor $2=$ identifying and understanding your own emotions, factor $3=$ dealing with emotions in others, and factor $4=$ perceiving emotion through facial expressions and body language. Correlations $\left(r_{\mathrm{s}}\right)$ between STEM and ESES factors are $-.007(\mathrm{p}=.921)$, .145 $(\mathrm{p}=.052)$, $.070(\mathrm{p}=.352)$, and $.025(\mathrm{p}=.736)$ for factors $1,2,3$, and 4 respectively. 
Running Head: Trajectories of first year university adjustment in a UK population 34

Table 5. Academic outcome by adjustment group.

\begin{tabular}{|c|c|c|c|c|c|c|c|c|}
\hline & \multicolumn{2}{|c|}{$\begin{array}{l}\text { Low, stable } \\
\text { adjustment }\end{array}$} & \multicolumn{2}{|c|}{$\begin{array}{c}\text { Medium, stable } \\
\text { adjustment }\end{array}$} & \multicolumn{2}{|c|}{$\begin{array}{l}\text { High, stable } \\
\text { adjustment }\end{array}$} & \multicolumn{2}{|c|}{$\begin{array}{c}\text { Low, increasing } \\
\text { adjustment }\end{array}$} \\
\hline \multirow[t]{2}{*}{$\mathrm{N}=$} & \multicolumn{2}{|c|}{102} & \multicolumn{2}{|c|}{128} & \multicolumn{2}{|c|}{73} & \multicolumn{2}{|c|}{28} \\
\hline & Mean & $\mathrm{SE}$ & Mean & $\mathrm{SE}$ & Mean & $\mathrm{SE}$ & Mean & $\mathrm{SE}$ \\
\hline $\begin{array}{l}\text { Academic } \\
\text { achievement* }\end{array}$ & 51.7 & 1.4 & 60.0 & 1.2 & 56.8 & 1.9 & 63.3 & 2.8 \\
\hline
\end{tabular}

Note. ${ }^{*}=$ Academic performance measured by end of year GPA. 
Running Head: Trajectories of first year university adjustment in a UK population 35

\section{Acknowledgments}

This research was funded by a grant from the Higher Education Academy (HEA) awarded to Tariq, Appleby, Barnes, and Qualter. Dr. Sian Roberts worked as a post-doc on that grant. 